\title{
Protoneutron stars and neutron stars
}

\author{
D. Gondek-Rosińska, P. Haensel and J. L. Zdunik \\ Nicolaus Copernicus Astronomical Center, Polish Academy of Sciences, \\ Bartycka 18, 00-716 Warszawa, Poland
}

\begin{abstract}
We find constraints on minimum and maximum mass of ordinary neutron stars imposed by their early evolution (protoneutron star stage). We calculate models of protoneutron stars using a realistic standard equation of state of hot, dense matter valid for both supranuclear and subnuclear densities. Results for different values of the nuclear incompressibility are presented.
\end{abstract}

\section{Introduction}

The newborn neutron star (protoneutron star - PNS) does not resemble the cold ordinary neutron star (NS). It is a very hot and lepton rich object. The minimum and the maximum allowable mass of PNSs are different than that corresponding to cold NSs. PNS evolves either to BH or to stable NS depending on its total number of baryons.

Constraints on $M_{\max }$ of NSs imposed by composition and equation of state (EOS) of hot dense stellar interior were studied by numerous authors (e.g. Takatsuka 1995, Bombaci et. al 1995, Bombaci 1996, Prakash et al. 1997). For low densities they took EOS of cold matter. Here we use a unified dense matter model, which holds for both supranuclear and subnuclear densities. We constrain $M_{\max }$ and $M_{\min }$ of NSs assuming conservation of the total baryon number of the star during its evolution. Since accretion on the forming protoneutron star ceases few seconds after core bounce (Chevalier 1989) we restrict ourselves to the late stage PNS (see Goussard et al. 1998, Strobel et al. 1999 for the discussion and definitions of the stages of PNS evolution); note that Strobel at al. (1999) considered PNS about 50-100 ms after core bounce as a initial stage. The problem of $M_{\min }$ of NS was studied by Gondek et al. 1998, but only for moderately stiff equation of state. Here we present results also for stiffer and softer equation of state.

\section{Calculations and results}

A few seconds after birth $t \simeq 2-4 \mathrm{~s}$ the matter in the core of a hot neutron star has almost constant lepton fraction $\left(Y_{l}=0.3-0.4\right)$ and entropy per baryon $(s=$ $1-2$ in unit of the Boltzmann constant $k_{B}$ ) (Burrows \& Lattimer 1986). Our models of PNS are composed of a hot, neutrino-opaque interior, and neutrinotransparent envelope. We find the position of the neutrinosphere in a selfconsistent way (for more details see Gondek et al. 1997). We consider the hot 
isentropic interior with $s=1$ or $s=2$ and a significant trapped lepton number $Y_{l}=0.4$. All calculations were done for a realistic, standard Lattimer-Swesty (1991) hot, dense matter model. To cover the present uncertainties of nuclear interaction and many-body technique, we construct models of PNS for three different values of the nuclear incompressibility; $K=375,220,180 \mathrm{MeV}$. We consider PNS as an isolated, non-rotating, spherically symmetric object, which has no magnetic field. Under these assumptions the structure of the star is solved numerically using TOV equations (Tolman 1939, Oppenheimer \& Volkoff 1939) for a given EOS.

The gravitational masses of NSs determined by maximum and minimum allowable masses of PNSs are smaller than $\sim 2.5 M_{\odot}$ and greater than $\sim 0.5 M_{\odot}$ (this is by a factor of ten larger than the minimum masses of static NSs sequences). The minimum baryon mass of PNSs at the earlier stages of their evolution was found to be 2-3 times larger (Goussard et al. 1998, Strobel et al. 1999 for the EOS of dense hot matter based on the Thomas-Fermi model) than in the stage considered by us. In this case a hot shocked stellar envelope has main influence on the minimum mass of PNS sequence.

We show that the maximum critical gravitational mass of a NS is by $\sim$ $0.14-0.19 M_{\odot}$ (depending on the nuclear incompressibility $K$ ) smaller than maximum mass of static NSs sequence. This result seems to be characteristic for a standard model of dense matter, composed of nucleons and leptons (e.g. Takatsuka 1995, Bombaci 1996). The conclusions are different when we take into account existence of an exotic components (hyperons, pions or kaons condensate or quark matter) at hight densities (Bombaci et al. 1996). Appearance of exotic matter could dramatically soften the EOS of dense matter lowering maximum allowable mass of NSs (e.g. Reddy et al. 1999). The deleptonization and cooling of PNSs of baryon mass exceeding the maximum allowable baryon mass for NSs, would lead to their collapse into BH.

Acknowledgments. This work was supported in part by the following grants KBN-2P03D01413 and KBN-2P03D02117.

\section{References}

Baumgarte T.W., Shapiro S.L., Teukolsky S.A., 1996, ApJ 458, 680

Bombaci I., Prakash M., Prakash M., Ellis P.J., Lattimer J.M., Brown G.E., 1995, Nucl. Phys. A, 583, 623

Bombaci I., 1996, A\&A 305, 871

Brown G.E., Bethe H.A., 1994, ApJ 423, 659

Burrows A., Lattimer J.M., 1986, ApJ, 307, 178

Chevalier R., 1989, ApJ, 346, 847

Gondek D., Haensel P., Zdunik J. L., 1997, A\&A, 325, 217

Gondek D., Haensel P., Zdunik J. L., 1998, PASP, 138, 131

Goussard J-O., Haensel P., Zdunik J. L., 1998, A\&A, 330, 1005

Lattimer J.M., Swesty F.D., 1991, Nucl. Phys. A535, 331

K. Strobel, C. Schaab, M. Weigel, 1999, A\&A, 350, 497

Takatsuka T., 1995, Nucl. Phys. A588, 365c 